RAMSKYi A., SAMARUK N., POPLAVSKA O.

\title{
THE DERIVATIVE CONNECTING PROBLEMS FOR SOME CLASSICAL POLYNOMIALS
}

Given two polynomial sets $\left\{P_{n}(x)\right\}_{n \geq 0}$, and $\left\{Q_{n}(x)\right\}_{n \geq 0}$ such that

$$
\operatorname{deg}\left(P_{n}(x)\right)=n, \operatorname{deg}\left(Q_{n}(x)\right)=n .
$$

The so-called the connecting problem between them asks to find the coefficients $\alpha_{n, k}$ in the expression $Q_{n}(x)=\sum_{k=0}^{n} \alpha_{n, k} P_{k}(x)$. Let $\left\{S_{n}(x)\right\}_{n \geq 0}$ be another polynomial set with $\operatorname{deg}\left(S_{n}(x)\right)=n$. The general connection problem between them consists in finding the coefficients $\alpha_{i, j}^{(n)}$ in the expansion

$$
Q_{n}(x)=\sum_{i, j=0}^{n} \alpha_{i, j}^{(n)} P_{i}(x) S_{j}(x)
$$

The connection problem for different types of polynomials has a long history, and it is still of interest. The connection coefficients play an important role in many problems in pure and applied mathematics, especially in combinatorics, mathematical physics and quantum chemical applications. For the particular case $Q_{n}(x)=P_{n+1}^{\prime}(x)$ the connection problem is called the derivative connecting problem and the general derivative connecting problem associated to $\left\{P_{n}(x)\right\}_{n \geq 0}$.

In this paper, we give a closed-form expression of the derivative connecting problems for wellknown systems of polynomials.

Key words and phrases: connection problem, inversion problem, derivative connecting problem, connecting coefficients, orthogonal polynomials.

Khmelnytskyi National University, 11 Instytytska str., 29016, Khmelnytskyi, Ukraine

E-mail: ramsky@ukr.net (Ramskyi A.), samaruk_nmeukr.net (Samaruk N.),

helen.poplavskaya@gmail. com (Poplavska O.)

\section{INTRODUCTION}

Given the two polynomial sets $\left\{P_{n}(x)\right\}_{n \geq 0},\left\{Q_{n}(x)\right\}_{n \geq 0}$ such that

$$
\operatorname{deg}\left(P_{n}(x)\right)=\operatorname{deg}\left(Q_{n}(x)\right)=n,
$$

for all $n$. The connection problem between them consists in finding the coefficients $\alpha_{n, k}$ in the expansion

$$
Q_{n}(x)=\sum_{k=0}^{n} \alpha_{n, k} P_{k}(x)
$$

$\mathrm{y} \Delta \mathrm{K} 519.1$

2010 Mathematics Subject Classification:33C05, 05A19. 
Let $\left\{S_{n}(x)\right\}_{n \geq 0}$ be another polynomial sets with $\operatorname{deg}\left(S_{n}(x)\right)=n$. The general connection problem between them consists in finding the coefficients $\alpha_{i, j}^{(n)}$ in the expansion

$$
Q_{n}(x)=\sum_{i, j=0}^{n} \alpha_{i, j}^{(n)} P_{i}(x) S_{j}(x) .
$$

For the particular case $Q_{n}(x)=P_{n+1}^{\prime}(x)$ the connection problem is called the derivative connecting problem and the general derivative connecting problem for the polynomial family $\left\{P_{n}(x)\right\}_{n \geq 0}$.

The study of such a problem has attracted a lot of interest in the last few years. For instance, the representations of parametric derivatives have been obtained by Froehlich [6] for Jacobi polynomials, by Koepf [7] for generalized Laguerre polynomials and Gegenbauer polynomials, by Koepf and Schmersau [8] for all the continuous and discrete classical orthogonal polynomials, in $[5,9,11,13]$ for classic orthogonal polynomials.

The derivative connecting problem is considered for Chebyshev polynomials of the first and the second types [10], for some Koornwinder polynomials in [1]. In [2,3] the derivation connection problem was solved for the Fibonacci, Lucas and Kravchuk polynomials and the authors use the solutions to produce new combinatorial identities for these polynomials. Also, the derivative connecting problem is solved in [4] for some hypergeometrical polynomials.

As an example let us consider the sequence of Appel polynomials $\left\{A_{n}(x)\right\}_{n \geq 0}$ with exponential generating function

$$
\mathcal{G}\left(A_{n}(x), z\right)=\mathcal{A}(z) e^{x z}=\sum_{n=0}^{\infty} A_{n}(x) \frac{z^{n}}{n !},
$$

where $\mathcal{A}(z)$ is an arbitrary formal power series, $\mathcal{A}(0) \neq 0$.

Then

$$
\frac{d}{d x} \mathcal{G}\left(A_{n}(x), z\right)=\mathcal{A}(z) e^{x z} z=\mathcal{G}\left(A_{n}(x), z\right) z=\sum_{n=0}^{\infty} A_{n}(x) \frac{z^{n+1}}{n !} .
$$

On the other side

$$
\frac{d}{d x} \mathcal{G}\left(A_{n}(x), z\right)=\frac{d}{d x} \sum_{n=0}^{\infty} A_{n}(x) \frac{z^{n}}{n !}=\sum_{n=0}^{\infty} A_{n}^{\prime}(x) \frac{z^{n}}{n !} .
$$

Equating the coefficients near $z^{n}$ we will find

$$
\frac{1}{n !} A_{n}(x)^{\prime}=\frac{1}{(n-1) !} A_{n-1}(x),
$$

and will obtain the solution of derivative connecting problem for Appel polynomials:

$$
A_{n}(x)^{\prime}=n A_{n-1}(x) .
$$

In the paper we solve these derivative connecting problems for many well-known classes of polynomials $P_{n}(x)$.

In Section 2, a general appearance of the decomposition of the derivative of the polynomial $P_{n}^{\prime}(x)$ is established, depending on the appearance of the logarithmic derivative of the generating function. In Section 3, the derivative connecting problem is solved for Lagguerre, Kravchuk, Charlier, Stirling, Bell, Bernoulii, Euler and Hermite polynomials. In Section 4, the general derivative connecting problem is solved for Chebyshev, Gegenbauer and Legendre polynomials. 


\section{The MAIN THEOREM}

We propose a method for solving the derivative connecting problem based on the use of the generating functions of polynomial families. The generation function of the family of polynomials $\left\{P_{n}(x)\right\}_{n \geq 0}$ is the formal functional series

$$
\mathcal{G}\left(P_{n}(x), z\right)=\sum_{n=0}^{\infty} c_{n} P_{n}(x) z^{n}
$$

where $c_{n}$ is a certain numerical sequence. For case of $c_{n}=1$ the generating function is called as ordinary generating function, and when $c_{n}=\frac{1}{n !}$ we obtain an exponential generating function.

Theorem 1. Let the logarithmic derivative of the ordinary generating function $\mathcal{G}\left(P_{n}(x), z\right)$ of the polynomials family $\left\{P_{n}(x)\right\}$ can be represented by the following series with rational coefficients

$$
\frac{d}{d x} \ln \mathcal{G}\left(P_{n}(x), z\right)=\sum_{i=1}^{\infty} a_{i} z^{i}
$$

Then

$$
P_{n}(x)^{\prime}=\sum_{i=1}^{n} a_{i} P_{n-i}(x)
$$

Let the logarithmic derivative of the exponential generating function $\mathcal{G}(x, z)$ of the polynomials family $\left\{P_{n}(x)\right\}$ is written as formal series with rational coefficients

$$
\frac{d \mathcal{G}(x)}{d x}=\sum_{i=1}^{\infty} a_{i} \frac{z^{i}}{n !}
$$

Then

$$
P_{n}(x)^{\prime}=\sum_{i=1}^{n} a_{i} \frac{n !}{(n-i) !} P_{n-i}(x)
$$

Proof. Assume that the generating function $\mathcal{G}\left(P_{n}(x), z\right)$ and its particular derivative $\mathcal{G}\left(P_{n}(x), z\right)_{x}^{\prime}$ are connected

$$
\mathcal{G}\left(P_{n}(x), z\right)_{x}^{\prime}=\mathcal{G}\left(P_{n}(x), z\right) R(z)
$$

where $R(z)=a_{1} z+a_{2} z^{2}+\cdots$ is a formal power series. Then

$$
\begin{gathered}
\mathcal{G}\left(P_{n}(x), z\right)_{x}^{\prime}=\sum_{n=0}^{\infty} c_{n} P_{n}^{\prime}(x) z^{n}=\left(\sum_{n=0}^{\infty} c_{n} P_{n}(x) z^{n}\right)\left(a_{1} z+a_{2} z^{2}+\cdots\right) \\
=\sum_{n=0}^{\infty}\left(\sum_{i=1}^{n} a_{i} c_{n-i} P_{n-i}(x)\right) z^{n} .
\end{gathered}
$$

Equating the coefficients at the same powers of $z$, we obtain that

$$
c_{n} P_{n}^{\prime}(x)=\sum_{i=1}^{n} a_{i} c_{n-i} P_{n-i}(x)=a_{1} c_{n-1} P_{n-1}+a_{2} c_{n-2} P_{n-2}+\cdots+a_{n} c_{0} P_{0}(x),
$$


which will be a solution of the derivative connection problem for the polynomial family $P_{n}(x)$.

For the case of the ordinary generating function, we have $c_{n}=1$ and so

$$
P_{n}^{\prime}(x)=\sum_{i=1}^{n} a_{i} P_{n-i}(x)
$$

Similarly, for the exponential generating function for $c_{n}=\frac{1}{n !}$ we obtain that

$$
P_{n}(x)^{\prime}=\sum_{i=1}^{n} a_{i} \frac{n !}{(n-i) !} P_{n-i}(x) .
$$

Proved theorem sets strict requirements for the generating function $\mathcal{G}\left(P_{n}(x), z\right)$-its logarithmic derivative must be a function of the one variable, although the generating function depends upon of two variables.

Suppose that the logarithmic derivative of the generating function is not a function of the variable and it has the following expansion

$$
\frac{d}{d x} \ln \mathcal{G}\left(P_{n}(x), z\right)=\sum_{i=1}^{\infty} a_{i}(x) z^{i}
$$

where $a_{i}(x)$ - some polynomial. In this case for polynomials $S_{n}(x)$ their degree is equal $n$ so they form the basis of the vector space of all polynomials from the variable $x$. Therefore the polynomials $a_{i}(x)$ can be expanded on this basis:

$$
a_{i}(x)=\sum_{j=0}^{i} \alpha_{i, j} S_{j}(x) .
$$

The following Theorem 1 may be proved similarly

Theorem 2. Let the logarithmic derivative of the generating function $\mathcal{G}\left(P_{n}(x), z\right)$ of the polynomials family $\left\{P_{n}(x)\right\}$ can be written as formal series

$$
\frac{d}{d x} \ln \mathcal{G}\left(P_{n}(x), z\right)=\sum_{i=1}^{\infty} a_{i}(x) z^{i}
$$

and

$$
a_{i}(x)=\sum_{j=0}^{i} \alpha_{i, j} S_{j}(x),
$$

for some coefficients $\alpha_{i, j}$. Then

$$
P_{n}(x)^{\prime}=\sum_{i=1}^{n} \sum_{j=0}^{i} \alpha_{i, j} Q_{j}(x) P_{n-i}(x) .
$$

\section{THE DERIVATIVE CONNECTING PROBLEM}

Let apply the proved theorems for solving of the derivative connecting problems for some types of the classical polynomials. 


\subsection{The Laguerre polynomials $L_{n}^{\lambda}(x)$}

The Laguerre polynomials are defined by the following formula

$$
L_{n}^{(\lambda)}(x)=\sum_{i=0}^{n}(-1)^{i}\left(\begin{array}{c}
n+\lambda \\
n-i
\end{array}\right) \frac{x^{i}}{i !}
$$

with ordinary generating function

$$
\mathcal{G}\left(L_{n}^{\lambda}(x), z\right)=(1-z)^{-\lambda-1} \mathrm{e}^{-\frac{x z}{1-z}}
$$

We find the derivatives by parameters

$$
\frac{d}{d x} \mathcal{G}\left(L_{n}^{\lambda}(x), z\right)=-(1-z)^{-2-\lambda} z \mathrm{e}^{-\frac{x z}{1-z}}=\frac{z \mathcal{G}\left(L_{n}^{\lambda}(x), z\right)}{z-1}
$$

and

$$
\frac{d}{d \lambda} \mathcal{G}\left(L_{n}^{\lambda}(x), z\right)=-(1-z)^{-\lambda-1} \mathrm{e}^{-\frac{x z}{1-z}} \ln (1-z) .
$$

Therefore, the logarithmic derivative has following form

$$
\begin{aligned}
& \frac{d}{d x} \ln \mathcal{G}\left(L_{n}^{\lambda}(x), z\right)=\frac{z}{z-1}=-\sum_{i=1}^{\infty} z^{i} \\
& \frac{d}{d \lambda} \ln \mathcal{G}\left(L_{n}^{\lambda}(x), z\right)=-\ln (1-z)=\sum_{i=1}^{\infty} \frac{1}{i} z^{i}
\end{aligned}
$$

so we proved the theorem:

\section{Theorem 3.}

$$
\begin{aligned}
& \frac{d}{d x} L_{n}^{\lambda}(x)=-\sum_{i=0}^{n-1} L_{i}^{\lambda}(x), \\
& \frac{d}{d \lambda} L_{n}^{\lambda}(x)=\sum_{i=1}^{n} \frac{1}{i} L_{n-i}^{\lambda}(x) .
\end{aligned}
$$

This coincides with results [8] and [13] obtained by other methods.

\subsection{The Kravchuk polynomials}

The Kravchuk polynomials are defined such formula

$$
K_{n}^{(p)}(x, N)=\sum_{j=0}^{n}(-1)^{j}(p-1)^{k-j}\left(\begin{array}{c}
x \\
j
\end{array}\right)\left(\begin{array}{c}
N-x \\
n-j
\end{array}\right),
$$

and have following generating function

$$
\mathcal{G}\left(K_{n}^{(p)}(x, N), z\right)=(1+(p-1) z)^{N-x}(1-z)^{x} .
$$




\section{Theorem 4.}

$$
\begin{aligned}
\frac{d}{d x} K_{n}^{(p)}(x, N) & =\sum_{i=1}^{n} \frac{(-1)^{i}(p-1)^{i}-1}{i} K_{n-i}^{(p)}(x, N), \\
\frac{d}{d N} K_{n}^{(p)}(x, N) & =\sum_{i=1}^{n} \frac{(-1)^{i+1}(p-1)^{i}}{i} K_{n-i}^{(p)}(x, N), \\
\frac{d}{d p} K_{n}^{(p)}(x, N) & =(N-x) \sum_{i=1}^{n}(-1)^{i-1}(p-1)^{i-1} K_{n-i}^{(p)}(x, N) .
\end{aligned}
$$

Proof. We find derivatives of the generating function for Kravchuk polynomials with respect to parameters $x, N, p$ :

$$
\begin{aligned}
\frac{d}{d x} \mathcal{G}\left(K_{n}^{(p)}(x, N), z\right) & =(1+(p-1) z)^{N-x}(1-z)^{x}(\ln (1-z)-\ln (1+(p-1) z)) \\
& =\mathcal{G}\left(K_{n}^{(p)}(x, N), z\right) \ln \left(\frac{1-z}{1+(p-1) z}\right), \\
\frac{d}{d N} \mathcal{G}\left(K_{n}^{(p)}(x, N), z\right) & =(1+(p-1) z)^{N-x} \ln (1+(p-1) z)(1-z)^{x}, \\
\frac{d}{d p} \mathcal{G}\left(K_{n}^{(p)}(x, N), z\right) & =\frac{(1+(p-1) z)^{N-x}(N-x) z(1-z)^{x}}{1+(p-1) z} .
\end{aligned}
$$

So

$$
\begin{aligned}
\frac{d}{d x} \mathcal{G}\left(K_{n}^{(p)}(x, N), z\right) & =\mathcal{G}\left(K_{n}^{(p)}(x, N), z\right) \ln \left(\frac{1-z}{1+(p-1) z}\right), \\
\frac{d}{d N} \mathcal{G}\left(K_{n}^{(p)}(x, N), z\right) & =\mathcal{G}\left(K_{n}^{(p)}(x, N), z\right) \ln (1+(p-1) z), \\
\frac{d}{d p} \mathcal{G}\left(K_{n}^{(p)}(x, N), z\right) & =\mathcal{G}\left(K_{n}^{(p)}(x, N), z\right) \frac{(N-x) z}{1+(p-1) z} .
\end{aligned}
$$

From here we find expansion of a logarithmic derivative in a formal series

$$
\begin{aligned}
\frac{d}{d x} \ln \mathcal{G}\left(K_{n}^{(p)}(x, N), z\right) & =\ln \left(\frac{1-z}{1+(p-1) z}\right)=\sum_{i=1}^{\infty} \frac{(-1)^{i}(p-1)^{i}-1}{i} z^{i}, \\
\frac{d}{d N} \ln \mathcal{G}\left(K_{n}^{(p)}(x, N), z\right) & =\ln (1+(p-1) z)=\sum_{i=1}^{\infty} \frac{(-1)^{i+1}(p-1)^{i}}{i} z^{i}, \\
\frac{d}{d p} \ln \mathcal{G}\left(K_{n}^{(p)}(x, N), z\right) & =\frac{(N-x) z}{1+(p-1) z}=(N-x) \sum_{i=1}^{\infty}(-1)^{i-1}(p-1)^{i-1} z^{i} .
\end{aligned}
$$

Applying the Theorem 1 we get the required result.

For a particular case $p=2$ the problem is solved in [3].

\subsection{The Charlier polynomials $c_{n}^{(a)}(x)$}

The Charlier polynomials $c_{n}^{(a)}(x)$ have such an exponential generating function

$$
\mathcal{G}\left(c_{n}^{(a)}(x), z\right)=\mathrm{e}^{z}\left(1-\frac{z}{a}\right)^{x} .
$$


From here it's easy to get that

$$
\begin{aligned}
& \frac{d}{d x} \ln \mathcal{G}\left(c_{n}^{(a)}(x), z\right)=\ln \left(1-\frac{z}{a}\right)=-\sum_{i=1}^{\infty} \frac{z^{i}}{i a^{i}} \\
& \frac{d}{d a} \ln \mathcal{G}\left(c_{n}^{(a)}(x), z\right)=\frac{x z}{a^{2}}\left(1-\frac{z}{a}\right)^{-1}=\sum_{i=1}^{\infty} \frac{x}{a^{i+1}} z^{i} .
\end{aligned}
$$

Therefore, the following theorem holds.

Theorem 5.

$$
\begin{aligned}
\frac{d}{d x} c_{n}^{(a)}(x) & =-\sum_{i=1}^{n} \frac{n !}{(n-i) ! i a^{i}} c_{n-i}^{(a)}(x), \\
\frac{d}{d a} c_{n}^{(a)}(x) & =a\left(c_{1}^{(a)}(x)-1\right) \sum_{i=1}^{n} \frac{n !}{(n-i) ! a^{i+1}} c_{n-i}^{(a)}(x) .
\end{aligned}
$$

\subsection{The Stirling and Bell polynomials.}

The Stirling and Bell polynomials $S_{n}(x)$ ( see [12]) are defined by the exponential generating function

$$
\left(\frac{z}{1-\mathrm{e}^{-z}}\right)^{x+1}=\sum_{i=0}^{\infty} S_{i}(x) \frac{z^{i}}{i !} .
$$

We have that the logarithmic derivatives is equal to

$$
\frac{d}{d x} \ln \left(\frac{z}{1-\mathrm{e}^{-z}}\right)^{x+1}=\ln \left(\frac{z}{1-\mathrm{e}^{-z}}\right) .
$$

Let's expand to series the function

$$
h(z)=\ln \left(\frac{z}{1-\mathrm{e}^{-z}}\right)
$$

preliminary differentiating it.

We have

$$
\begin{aligned}
& \frac{d}{d z}(\ln (h(z)))=\frac{h^{\prime}(z)}{h(z)}=\frac{1}{z}-\frac{e^{-z}}{1-e^{-z}}=\frac{e^{z}-1-z}{z^{2}} \cdot \frac{z}{e^{z}-1}=\sum_{n=0}^{\infty} \frac{z^{n}}{(n+2) !} \cdot \sum_{n=0}^{\infty} \frac{B_{n} z^{n}}{n !} \\
& =\sum_{n=0}^{\infty}\left(\sum_{k=0}^{n} \frac{B_{k}}{(n-i+2) ! i !}\right) z^{n}=\sum_{n=0}^{\infty} \frac{z^{n}}{(n+2) !} \sum_{k=0}^{n}\left(\begin{array}{c}
n+2 \\
k
\end{array}\right) B_{k}=\frac{1}{2}-\sum_{n=1}^{\infty} \frac{B_{n+1}}{(n+1) !} z^{n} .
\end{aligned}
$$

Here we used the known identity

$$
\sum_{i=0}^{n-1}\left(\begin{array}{l}
n \\
i
\end{array}\right) B_{i}=0
$$

and the fact that the generating function for the Bernoulli numbers $B_{i}$ is equal to

$$
\frac{z}{\mathrm{e}^{z}-1}=\sum_{i=1}^{n} B_{i} \frac{z^{i}}{i !} .
$$

Note that the function $h(z)$ has a removable gap point at $z=0$ and

$$
h(0)=\lim _{z \rightarrow 0} h(z)=\lim _{z \rightarrow 0} \frac{1}{\left(1-\mathrm{e}^{-z}\right)^{\prime}}=1 .
$$


Therefore, by integrating, taking into account that $h(0)=1$, we get

$$
h(z)=\int\left(\frac{1}{2}-\sum_{n=1}^{\infty} \frac{B_{n+1}}{(n+1) !} z^{n}\right) d z=\frac{z}{2}-\sum_{n=1}^{\infty} \frac{B_{n+1}}{n(n+1) !} z^{n+1} .
$$

Consequently, we have proved the theorem.

Theorem 6.

$$
\frac{d}{d x} S_{n}(x)=\sum_{i=1}^{n}\left(\begin{array}{l}
n \\
i
\end{array}\right) \frac{B_{i}}{i} S_{n-i}(x) .
$$

The Bell polynomials $\varphi_{n}(x)$ are determined through the Stirling numbers of second type

$$
\varphi_{n}(x)=\sum_{i=0}^{n} S(n, i) x^{n}
$$

and have the generating function

$$
e^{x\left(e^{z}-1\right)}
$$

In the same way as in the case of Stirling polynomials the following statement is proved.

\section{Theorem 7.}

$$
\frac{d}{d x} \varphi_{n}(x)=\sum_{i=1}^{n}\left(\begin{array}{l}
n \\
i
\end{array}\right) \varphi_{n-i}(x) .
$$

\subsection{Generalized Bernoulli, Euler and Hermite polynomials}

Generalized Bernoulli $B_{n}^{(a)}(x)$, Euler $E_{n}^{(a)}(x)$ and Hermite $H_{n}^{(a)}(x)$ polynomials are defined by the following exponential generating function

$$
\begin{aligned}
e^{x z}\left(\frac{z}{e^{z}-1}\right)^{a} & =\sum_{i=0}^{\infty} B_{n}^{(a)}(x) \frac{z^{n}}{n !}, \\
e^{x z}\left(\frac{2}{e^{z}+1}\right)^{a} & =\sum_{i=0}^{\infty} E_{n}^{(a)}(x) \frac{z^{n}}{n !}, \\
e^{x z} e^{-a t^{2}} & =\sum_{i=0}^{\infty} H_{n}^{(a)}(x) \frac{z^{n}}{n !} .
\end{aligned}
$$

With respect to the variable $x$ these polynomials are the Appel polynomials, see [12], therefore for all three types of polynomials the following is performed

$$
\begin{aligned}
& \frac{d}{d x} B_{n}^{(a)}(x)=n B_{n-1}^{(a)}(x), \\
& \frac{d}{d x} E_{n}^{(a)}(x)=n E_{n-1}^{(a)}(x), \\
& \frac{d}{d x} H_{n}^{(a)}(x)=n H_{n-1}^{(a)}(x) .
\end{aligned}
$$

Let's find the logarithmic derivatives by parameter $a$ :

$$
\begin{aligned}
& \frac{d}{d a} \ln B_{n}^{(a)}(x)=\ln \left(\frac{z}{e^{z}-1}\right)=-\frac{z}{2}+(-1)^{n+1} \sum_{i=2}^{\infty} \frac{B_{i}}{i \cdot i !} z^{i}, \\
& \frac{d}{d a} \ln E_{n}^{(a)}(x)=\ln \left(\frac{2}{\mathrm{e}^{z}+1}\right)=\frac{1}{2} \sum_{i=1}^{n} \frac{E_{i-1}(1)}{i !} z^{i}, \\
& \frac{d}{d a} \ln H_{n}^{(a)}(x)=-z^{2},
\end{aligned}
$$


here $B_{i}, E_{i}(1)$ - are the Bernoulli numbers and Euler numbers respectively. Expansion

$$
\ln \left(\frac{z}{e^{z}-1}\right)=-\frac{z}{2}+\sum_{i=2}^{\infty} \frac{(-1)^{n+1} B_{i}}{i \cdot i !} z^{i}
$$

is obtained in the same way as expansion in subsection 2.4.

So, the following statement takes place.

\section{Theorem 8.}

$$
\begin{aligned}
\frac{d}{d a} B_{n}^{(a)}(x) & =-\frac{n}{2} B_{n-1}^{(a)}(x)+\sum_{i=2}^{n} \frac{(-1)^{n+1} B_{i}}{i}\left(\begin{array}{c}
n \\
i
\end{array}\right) B_{n-i}^{(a)}(x), \\
\frac{d}{d a} E_{n}^{(a)}(x) & =\frac{1}{2} \sum_{i=1}^{n}\left(\begin{array}{c}
n \\
i
\end{array}\right) E_{i-1}(1) E_{n-i}^{(a)}(x), \\
\frac{d}{d a} H_{n}^{(a)}(x) & =-n(n-1) H_{n-2}^{(a)}(x) .
\end{aligned}
$$

\section{A GENERALIZED DERIVATIVE CONNECTING PROBLEM}

\subsection{The Chebyshev polynomials}

The Chebyshev polynomials $T_{n}(x)$ of the first kind and the Chebyshev polynomials $U_{n}(x)$ of the second kind are determined by such ordinary generating function

$$
\mathcal{G}\left(T_{n}(x), z\right)=\frac{1-x z}{1-2 x z+z^{2}}, \quad \mathcal{G}\left(U_{n}(x), z\right)=\frac{1}{1-2 x z+z^{2}} .
$$

The following theorem take place.

\section{Theorem 9.}

$$
\begin{aligned}
& \frac{d}{d x} T_{n}(x)=T_{0}(x) T_{n-1}(x)+3 T_{1}(x) T_{n-2}(x) \\
& +\sum_{i=3}^{\infty}\left(T_{0}(x) T_{1}(x)^{i-1}+2 \sum_{k=1}^{i-1} T_{k}(x) T_{1}(x)^{i-1-k}\right) T_{n-i}(x), \\
& \frac{d}{d x} U_{n}(x)=2 \sum_{i=1}^{n} U_{i-1}(x) U_{n-1-i}(x) .
\end{aligned}
$$

Proof. We have

$$
\begin{aligned}
& \frac{d}{d x} \ln \mathcal{G}\left(T_{n}(x), z\right)=\frac{z\left(z^{2}-1\right)}{(1-x z)\left(1-2 x z+z^{2}\right)} \\
& =T_{0}(x) z+3 T_{1}(x) z^{2}+\sum_{i=3}^{\infty}\left(T_{0}(x) T_{1}(x)^{i-1}+2 \sum_{k=1}^{i-1} T_{k}(x) T_{1}(x)^{i-1-k}\right) z^{i}
\end{aligned}
$$

For the Chebyshev polynomials $U_{n}(x)$ of the second kind we have

$$
\frac{d}{d x} \ln \mathcal{G}\left(U_{n}(x), z\right)=\frac{2 z}{1-2 x z+z^{2}}=2 \sum_{i=1}^{\infty} U_{i-1}(x) z^{i}
$$

Therefore

$$
U_{n}(x)^{\prime}=2 \sum_{i=1}^{n} U_{i-1}(x) U_{n-1-i}(x)
$$




\subsection{The Gegenbauer and Legendre polynomials}

The Gegenbauer polynomials $C_{n}^{\lambda}(x)$ are determined by ordinary generating function

$$
\mathcal{G}\left(C_{n}^{\lambda}(x), z\right)=\frac{1}{\left(1-2 x z+z^{2}\right)^{\lambda}}
$$

It is logarithmic derivative is expressed through Chebyshev polynomials

$$
\begin{aligned}
\frac{d}{d x} \ln \mathcal{G}\left(C_{n}^{\lambda}(x), z\right) & =\frac{2 \lambda z}{1-2 x z+z^{2}}=2 \lambda \sum_{i=1}^{\infty} U_{i-1}(x) z^{i}, \\
\frac{d}{d \lambda} \ln \mathcal{G}\left(C_{n}^{\lambda}(x), z\right) & =-\ln \left(1-2 x z+z^{2}\right)=2 \sum_{i=1}^{\infty} \frac{1}{i} T_{i}(x) z^{i} .
\end{aligned}
$$

\section{Theorem 10.}

$$
\begin{aligned}
\frac{d}{d x} C_{n}^{\lambda}(x) & =2 \lambda \sum_{i=1}^{\infty} U_{i-1}(x) C_{n-i}^{\lambda}(x), \\
\frac{d}{d \lambda} C_{n}^{\lambda}(x) & =2 \sum_{i=1}^{n} \frac{1}{i} T_{i}(x) C_{n-i}^{(\lambda)}(x) .
\end{aligned}
$$

In [13] another expressions for the Gegenbauer polynomials were obtained. The Legendre polynomials $P_{n}(x)$ are determined by generating function

$$
\mathcal{G}\left(P_{n}(x), z\right)=\frac{1}{\sqrt{1-2 x z+z^{2}}}
$$

We have

$$
\frac{d}{d x} \ln \mathcal{G}\left(P_{n}(x), z\right)=\frac{z}{1-2 x z+z^{2}}=\sum_{i=1}^{\infty} U_{i-1}(x) z^{i} .
$$

Therefore there is the following assertion.

\section{Theorem 11.}

$$
\frac{d}{d x} P_{n}(x)=\sum_{i=1}^{\infty} U_{i-1}(x) P_{n-i}(x)
$$

\section{REFERENCES}

[1] Aktas R. Representations for parameter derivatives of some Koornwinder polynomials in two variables. J. Egyptian Math. Soc. 2016, 24, 555-561

[2] Bedratyuk L. Derivations and Identitites for Fibonacci and Lucas Polynomials. Fibonacci Quart. 2013, 51 (4), 351366.

[3] Bedratyuk L. Derivations and Identities for Kravchuk Polynomials. Ukrainian Math. J. 2014, 65 (12), $1755-1773$. doi:10.1007/s11253-014-0896-9

[4] Bedratyuk L., Bedratuyk A. The inverse and derivative connecting problems for some Hypergeometric polynomials. Carpathian Math. Publ. 2018, 10 (2), 235-247. doi:10.15330/cmp.10.2.235-247

[5] Bonan S., Lubinsky D., Nevai P. Orthogonal polynomials and their derivatives II. SLAM J. Math. Anal. 1987, 18, 1163-1176.

[6] Fröhlich J. Parameter derivatives of the Jacobi polynomials and the Gaussian hypergeometric function. Integral Transforms Spec. Funct. 1994, 2, 252-266. doi:10.1080/10652469408819056 
[7] Koepf W. Identities for families of orthogonal polynomials and special functions. Integral Transforms Spec. Funct. 1997, 5 (1-2), 69-102.

[8] Koepf W., Schmersau D. Representations of orthogonal polynomials. J. Comput. Appl. Math. 1998, 90, 57-94. doi:10.1016/S0377-0427(98)00023-5

[9] Lewanowicz S. Representations for the parameter derivatives of the classical orthogonal polynomials Rend. Circ. Mat. Palermo, Ser. II, Suppl. 2002 68, 599-613.

[10] Prodinger H. Representing derivatives of Chebyshev polynomials by Chebyshev polynomials and related questions. Open Math. 2017, 15, 1156-1160.

[11] Ronveaux A., Zarzo A., Area I., Godoy E. Classical orthogonal polynomials: dependence on parameters J.Comput. Appl. Math. 2000, 121, 95-112

[12] Steven Roman. The umbral calculus. Academic Press, 1983.

[13] Szmytkowski R. A note on parameter derivatives of classical orthogonal polynomials. arXiv:0901.2639v3.

Received 28.02.2019

Рамський А.О., Самарук Н.М., Поплавська О.А. Задачі диферениіальної зв'язності для деяких класииних многочленів // Карпатські матем. публ. - 2019. - Т.11, №2. - С. 431-441.

Нехай дано дві множини многочленів $\left\{P_{n}(x)\right\}_{n \geq 0}$ та $\left\{Q_{n}(x)\right\}_{n \geq 0}$ таких, що

$$
\operatorname{deg}\left(P_{n}(x)\right)=n, \operatorname{deg}\left(Q_{n}(x)\right)=n .
$$

Так звана задача диференціальної зв' язності між ними полягає у знаходженні коефіцієнтів $\alpha_{n, k}$ у виразі $Q_{n}(x)=\sum_{k=0}^{n} \alpha_{n, k} P_{k}(x)$.

Нехай $\left\{S_{n}(x)\right\}_{n \geq 0}$ - це інша множина порядку $\operatorname{deg}\left(S_{n}(x)\right)=n$. Узагальнена задача зв' язності між ними полягає у знаходженні коефіцієнтів $\alpha_{i, j}^{(n)}$ у виразі

$$
Q_{n}(x)=\sum_{i, j=0}^{n} \alpha_{i, j}^{(n)} P_{i}(x) S_{j}(x) .
$$

Задача зв'язності для різних типів многочленів має довгу історію, проте залишається цікавою і тепер. Коефіцієнти зв' язності грають важливу роль у багатьох задачах класичної та прикладної математики, особливо в комбінаториці, а також у математичній фізиці та прикладних застосуваннях квантової хімії. Аля часткового випадку, коли $Q_{n}(x)=P_{n+1}^{\prime}(x)$, задачу зв'язності називають диференціальною задачею зв'язності і відносять ії до множини $\left\{P_{n}(x)\right\}_{n \geq 0}$.

У статті наведено вирази у замкнутій формі задач диференціальної зв' язності для відомих систем многочленів.

Ключові слова і фрази: задача зв' язності, обернена задача, задача диференціальної зв' язності, коефіцієнти звя'зності, гіпергеометричні функції, гіпергеометричні многочлени. 